\title{
TÉMOIGNAGES
}

\section{Partenariat et formation à distance à la recherche}

Quelques indications de départ sont nécessaires en introduction à ce témoignage qui pose à la fois des problèmes de partenariat, de formation à distance et de recherche. Le département des Sciences du langage et de la communication de l'université de Rouen avait initié en 1989 une coopération avec diverses universités vietnamiennes dans le domaine de la formation des enseignants. Durant les longues années de guerre, la question de la formation n'avait pu constituer une priorité pour le pays, tout particulièrement sur le plan des formations en langues étrangères. Les formations à distance sont alors apparues comme une réponse pour combler dans l'immédiat un manque et le partenariat ainsi instauré comme un dispositif voué à terme à évoluer. Ce partenariat a eu, dès ses premiers balbutiements, une dimension éthique et politique. Les enseignants-chercheurs qui s'étaient engagés dans cette aventure, avaient en tête de mettre leurs compétences au service d'un pays qui avait besoin de se reconstruire et de se développer. Les premières collaborations ont eu cet enthousiasme partagé pour cadre et les premières rencontres une reconnaissance idéologique et scientifique réciproque comme arrière-fond. Et puis, des besoins ont disparu, d'autres se sont affinés, précisés, diversifiés, d'autres enfin sont nés de plus d'une décennie de collaboration.

\section{Des formations à distance de deuxième et troisième cycles}

Cette action, soutenue par le ministère des Affaires étrangères, a été étendue à d'autres pays de la zone (Laos, Cambodge, Chine). Elle avait pris deux formes principales : a) une formation par télé-enseignement, appuyée par deux missions annuelles sur site, b) l'accueil à l'université de Rouen de boursiers vietnamiens. Les formations proposées concernaient la licence et la maîtrise. Les étudiants étaient soit des universitaires professeurs de français, soit des universitaires d'enseignements spécialisés (médecine, architecture, etc.), soit des enseignants dans les domaines de l'interprétariat et de la traduction, soit des professeurs de français langue étrangère $\mathrm{du}$ secondaire, en particulier intervenant dans des filières bilingues (enseignement du français et en français), soit des étudiants en fin de cursus universitaire de français.

En 1991, un DEA de Sciences du langage est habilité à l'université de Rouen, sous l'intitulé Langage en situation. Il peut être préparé en télé-enseignement. Étant le seul de ce type en France dans cette discipline, il attire de nombreuses candidatures nationales et internationales, en particulier des enseignants étrangers 
dans le cadre des conventions soutenues financièrement par le MAE. Ceux-ci préparent ce diplôme par télé-enseignement ou comme boursiers à Rouen, les soutenances se faisant soit sur site, soit à Rouen. La recherche peut se poursuivre ensuite en thèse au sein du laboratoire "Dynamiques Socio-langagières » (DYALANG, UMR 6065) dans le cadre de l'école doctorale en SHS «Savoirs, critique, expertises » de l'université de Rouen. Le doctorat se fait alors à distance avec deux séjours à Rouen : deux mois en début de thèse pour la documentation et huit mois en fin de thèse pour la rédaction finale et la soutenance. Sur un plan très général, ces formations à distance ont eu, au départ, pour premier objectif de donner l'occasion à nos collègues asiatiques d'obtenir des diplômes universitaires de haut niveau (en particulier des doctorats français), qui leur ont ainsi permis de faire institutionnellement reconnaître leurs compétences en français, en didactique, en linguistique. Puis, des intérêts scientifiques communs sont venus se greffer sur les premières motivations et ont fait évoluer la nature même du partenariat.

Les premiers supports utilisés ont été les polycopiés de cours, avec un suivi entre enseignants et étudiants par courrier classique et par télécopie. L'envoi de cassettes audio et vidéo a constitué une première amélioration pour la diffusion de la formation et pour le contact entre les acteurs. Mais c'est la récente mise en ligne de tous les cours, la création d'un campus numérique et le projet de vidéo-conférences, ainsi que les messageries électroniques qui ont complètement modifié la nature du partenariat par une interactivité inédite et une individualisation de la formation, coûteuse en temps pour les enseignants, mais d'une grande efficacité pour la qualité de la construction des savoirs.

Cependant, à côté de ces évolutions technologiques majeures, une relation directe et régulière avec les étudiants est apparue indispensable. C'est à l'intention des étudiants d'Asie du Sud-Est de troisième cycle que notre partenariat a mis en place une formation à la recherche « décentralisée ». En effet, les missions financées par le MAE étaient, d'une part, uniquement locales (au Vietnam, au Laos ou au Cambodge), et, d'autre part, essentiellement orientées en direction des étudiants des niveaux licence et maîtrise. Il était donc devenu urgent d'encadrer spécifiquement les inscrits en DEA (nécessité d'une formation méthodologique en présentiel) et les inscrits en thèse (difficulté due à l'éloignement du directeur de recherche).

La présence d'un grand nombre d'étudiants résidant en Asie du Sud-Est, inscrits à Rouen dans le cadre des conventions inter-universitaires, a conduit à la création d'une formation doctorale décentralisée, intitulée «Dynamiques francophones en Asie du Sud-Est». Cette formation, qui comporte chaque année une soixantaine d'étudiants en direction de recherche dans le laboratoire DYALANG, a obtenu une aide de l'AUPELF-UREF (l'actuelle AUF) pour financer un séminaire annuel de méthodologie et d'actualisation des connaissances. Ce séminaire doctoral réunit, depuis 1998, dans l'une des universités partenaires, l'ensemble des étudiants de thèse et de DEA des divers pays de la zone. 


\section{Une formation doctorale en Asie du Sud-Est}

Les objectifs de cette formation en termes de recherche sont divers et ont évolué au fil des années. Ils sont présentés ici dans un ordre chronologique qui montre le point de départ FLE et l'évolution vers des objectifs diversifiés, plus conformes à la fois aux besoins réels de nos partenaires et à nos intérêts propres de recherche et de formation à la recherche en sciences du langage. Il s'agit des points suivants.

1) Accélérer la production de thèses essentiellement centrées sur le français en Asie du Sud-Est à un moment critique, tant pour le devenir de ces pays que pour la place que le français joue et jouera dans ces évolutions :

- analyse des problèmes spécifiques que pose l'apprentissage de la langue française ;

- analyse des problèmes d'appropriation de certaines pratiques langagières (par exemple et en particulier: la lecture en français, la planification textuelle, la communication dans des situations formelles, etc.) ;

- analyse des situations de contact des langues et des cultures entre le vietnamien et le français (approches historiques et socio-linguistiques, perspectives interculturelles) ;

- description des usages du français dans les divers domaines de la coopération scientifique et technique et analyse des besoins de formation, voire d'équipement linguistique, de standardisation ;

- analyse des problèmes posés par l'usage du français comme langue d'enseignement (dans les classes bilingues mises en place au Vietnam notamment) ;

- mises au point de didactiques spécifiques (formations à objectifs spécifiques, langues de spécialité, commerce, tourisme, vulgarisation scientifique et technique);

- analyse du statut du français en Asie du Sud-Est en relation avec la problématique de la francophonie (en liaison avec l'Agence universitaire francophone).

2) Former de bons analystes de discours pour faire face aux besoins (essentiellement en terminologie) de modernisation de la langue vietnamienne (dans les domaines du commerce, de la santé, de la justice, etc.).

3) Contribuer au développement d'une école doctorale francophone de la zone, avec pour base humaine d'encadrement et de tutorat les docteurs déjà formés (c'està-dire, aujourd'hui, environ une trentaine de personnes); cette école aura pour objectif d'assurer en matière de formation ce qui est exigé de l'école doctorale SHS de l'université de Rouen qui, elle, ne délivre pas de formation à distance.

4) Utiliser au mieux les moyens financiers et humains pour développer des synergies sur la base de coopérations bilatérales en vue de collaborations de recherche et d'aide à la création de laboratoires de recherche ; ce qui signifie aussi d'aider à la formation d'enseignants-chercheurs habilités à diriger des recherches. 
Mais, les années passant, l'équipe organisatrice se transforme, les docteurs de plus en plus nombreux venant renforcer l'encadrement du stage. Puis, des conférenciers d'universités francophones sont invités pour présenter leurs travaux. Le séminaire se complexifie en diversifiant les participants. Aux directeurs de recherche rouennais et à leurs étudiants, viennent s'ajouter les nouveaux docteurs et des chercheurs extérieurs. A côté des ateliers de direction de recherche, se multiplient des plages de communications en séminaire. La manière de désigner ce rassemblement de recherche évolue aussi : appelé stage, puis séminaire, il se désigne aujourd'hui comme colloque.

Une telle évolution a posé de nombreux problèmes de fond qui vont être présentés et problématisés dans les lignes qui suivent, car l'expérience a de multiples facettes.

Dans les limites de cette contribution, quelques notions, qui se sont révélées centrales parce qu'éclairantes, seront exposées: celles de besoins, d'objectifs, d'autonomie et de savoir.

\section{Partenariat et analyse des besoins}

Cette problématique fait partie de notre méthodologie de partenariat de manière à instaurer un cheminement commun de recherche, évolutif et efficace. Une différence s'impose immédiatement entre besoins réels et besoins ressentis de recherche. Ces derniers prennent d'emblée le pas sur les autres, car ce que nos partenaires visent en premier lieu, ce sont des « espérances pratiques » d'action immédiate sur le terrain, tel qu'il est vécu au quotidien sans prise de distance, sans analyse. Bien évidemment, on doit y répondre dans un premier temps. Mais la collaboration ne peut réussir que si un deuxième temps se dessine qui comprend deux aspects. Premier aspect : répondre aux demandes de recherche exige, à un moment donné de la collaboration, que celles-ci soient construites sur le long terme et fondées en vue de définir des besoins réels. Second aspect : faire connaître nos offres de recherche, à partir desquelles nos partenaires se situeront, s'impose aussi. En effet, les besoins dits objectifs sont très souvent ceux que l'on ne ressent pas, mais qui pourtant existent bel et bien. Ainsi, nos offres permettent à nos partenaires à la fois de prélever ce qui leur convient et de découvrir des pistes utiles auxquelles ils n'avaient pas pensé. Cette rencontre entre demandes et offres a une fonction essentielle dans le partenariat : elle limite le poids des représentations sociales, fortes de part et d'autre, et permet une réelle construction commune des besoins de recherche.

Mais il faut bien se garder de croire que l'analyse des besoins est une démarche que l'on met en place une seule fois, et une fois pour toutes, dans le partenariat. La notion de besoin évolue toute au long de la collaboration par la dynamique même de collaboration et par l'inévitable déplacement des enjeux pour y répondre. On sait bien que celui qui se forme change au fur et à mesure qu'il apprend et que celui qui forme modifie son niveau d'exigence. Citant Pierre Bourdieu, Louis Porcher 
rappelle que l'apprenant, dès qu'il a véritablement appris quelque chose, qu'il l'a profondément assimilé, oublie qu'il l'a appris (voir le numéro spécial du Français dans le monde, Recherches et applications de 1992). On ne doit surtout pas négliger dans un partenariat de formation cette « amnésie des apprentissages » qui témoigne d'une «incorporation réussie». Ainsi, tout au long de la formation, de nouveaux souhaits apparaissent, des désirs inédits sont formulés, d'anciennes nécessités deviennent caduques et les espérances pratiques elles-mêmes se transforment. C'est pourquoi il est raisonnable que l'analyse des besoins soit reconnue comme une opération à réitérer de façon régulière au cours du partenariat. Il s'agit en effet d'un indispensable outil de régulation.

\section{Partenariat et objectifs de formation}

De plus, cette analyse des besoins permet de connaître le public auquel on a affaire, aspect nécessaire pour construire des objectifs de formation à la recherche adaptés au terrain. La collaboration consiste en effet à mettre à l'épreuve d'une situation particulière le métier de chercheur. Il y a là une rencontre à bâtir entre des savoir-faire scientifiques, universellement partagés, et des habitudes culturelles, de part et d'autre spécifiques. C'est dire que l'offre existe avant la demande, mais que, par le biais de la connaissance des besoins et du terrain, elle se modifie et s'adapte.

Il existe donc, dans un partenariat de formation réussi, un perpétuel mouvement de va-et-vient entre l'analyse des besoins de recherche et la détermination des objectifs de recherche. Ce double mouvement montre que l'on peut soit partir d'objectifs de recherche pour aller vers les besoins, soit l'inverse, les deux mouvements étant dialectiquement liés. Mais, dans les deux cas, la négociation n'est pas la même, le pouvoir n'est pas du même côté. Dans le premier cas, c'est celui qui offre qui impose sa démarche, dans le second, c'est le demandeur qui impose la sienne. Dans ce domaine, un équilibre est indispensable pour réguler les inévitables zones de conflit et pour gérer les prévisibles tensions.

Voici des exemples de ces deux mouvements dans le choix de la thématique de thèse. Deux cas dans lesquels les besoins du terrain demandeur ont rejoint des objectifs de recherche disciplinaire: Monsieur Pham Duc Su, «Statut de la traduction dans la pratique de lecture en langue étrangère » et Madame Nguyen Kim Oanh, « Contrat didactique et discours professoral en classe de langue ».

Deux cas pour lesquels ce sont les objectifs de la recherche disciplinaire qui ont révélé des besoins de terrain : Monsieur Le Viet Dung, «Prise de parole et identité : un questionnement socio-linguistique sur la pratique langagière quotidienne des Vietnamiens » et Madame Nguyen Thi Ngoc Suong, «Cohérence dans le discours économique : le cas du papier d'analyse dans la presse écrite au Vietnam ». 


\section{Partenariat et autonomie}

Le partenariat de recherche vise la lente disparition de l'inégalité d'expertise entre les partenaires et donc une prise d'autonomie dans la recherche des formés. L'autonomie, on le sait, est la capacité de l'apprenant à conduire sans aide son activité propre, à être à lui-même sa loi (auto-nomos), à décider sans dépendre de qui que ce soit et des besoins et des objectifs. L'autonomie n'est pas une revanche sur l'autre, mais le but même de tout apprentissage, la condition expresse d'une collaboration réussie. Sinon, la dynamique est rompue et la relation, fossilisée dans un statut quo inopérant, n'a plus lieu d'être.

Mais l'autonomie ne se décrète pas un beau matin! Il s'agit d'un lent processus d'autonomisation qui exige là aussi un double mouvement. D'une part, que toute tentation d'hétéronomie ait disparu du côté des formés (on ne lâche pas facilement la main de celui qui nous a conduits). D'autre part, que l'expert accepte de perdre une forme de pouvoir et change de statut dans la collaboration, c'est-à-dire devienne un «pair» dans la recherche. Cette évolution s'apprend et, sur ce plan, les partenaires sont autant l'un que l'autre en situation d'apprentissage. Il s'agit de grandir ensemble, de progresser vers cette autonomie que l'on a co-construite; il s'agit donc d'une démarche de professionnalisation de part et d'autre, recherchée, voulue dès le départ de la collaboration, mais dont on n'avait pas exactement conscience ou que l'on ne voulait pas vraiment voir. Cette démarche modifie les statuts et rôles des partenaires et devient l'objet, à son tour, de négociations. Jusqu'à quand l'ancien expert demeure-t-il un recours? A partir de quand le nouvel expert devient-il un collaborateur? Cette aptitude à la négociation des rôles est au cœur même du processus d'autonomisation et marque la responsabilité de chacun dans le partenariat.

Voici, pour exemple, le parcours exemplaire d'un de nos étudiants vietnamiens. Monsieur Tran Duc Tuan est venu à Rouen en 1995 sur un projet d'enseignement de FLE en langue de spécialité. Il a soutenu en 1996 un DEA La terminologie médicale vietnamienne, suivi en 1999 par la soutenance d'un excellent doctorat La standardisation de la terminologie médicale vietnamienne: une approche socioterminologique. La recherche porte sur le vocabulaire médical orienté vers son apprentissage au Vietnam et sa traduction en français. Le dictionnaire, préfiguré dans la thèse, comprend des analyses sémantiques et contextuelles dans les deux langues. Ce travail lui a valu l'attribution par l'AUF d'une bourse post-doctorale pour un séjour à l'Université Laval au Québec. Monsieur Tran Duc Tuan est, depuis, l'auteur de deux lexiques très usités et de plusieurs articles au Vietnam. Il vient d'être recruté par le laboratoire d'informatique médicale du CHUR de Rennes. Une collaboration de recherche est prévue avec notre laboratoire lorsqu'il reviendra au Vietnam (codirections de thèses et poursuite de travaux de politique linguistique et de standardisation terminologique). 


\section{Partenariat et savoir autonome}

Seuls les savoirs qui conduisent à l'autonomie permettent la poursuite des partenariats, parce qu'ils font partie de l'identité des apprenants et peuvent être réinvestis dans la recherche à égalité de part et d'autre.

Nous concernant, la première étape de cette autonomie a été la création de relais locaux dans le suivi des étudiants de troisième cycle. Sont ainsi devenus « tuteurs » pour les nouveaux inscrits les diplômés de l'université de Rouen revenus dans leur pays d'origine. Ces docteurs ont donc été directement associés au fonctionnement même du partenariat en constituant sur place un accueil des étudiants et une structure de séminaires de discussion et d'échange. Dans une deuxième étape, et en relation avec les directeurs de recherche français, ils ont assuré les soutenances de DEA, sous l'autorité d'un directeur de recherche français, en mission pour la session d'examens. Cette solution, qui économise la venue en nombre d'étudiants asiatiques en France pour l'envoi d'un seul directeur de recherche sur place, confère une responsabilité scientifique nouvelle à nos partenaires et un pouvoir qui, dans une troisième étape, va nous conduire vers un système de codirections d'abord et de cotutelles ensuite.

En effet, la codirection de thèse prévoit deux directeurs, un sur place (docteur et universitaire) et un français (habilité à diriger des recherches). La cotutelle, quant à elle, suppose deux exigences nouvelles : la création sur place d'équipes de recherche reconnues comme équipes d'accueil des étudiants et des collègues asiatiques habilités à diriger des recherches par une procédure reconnue en France. Ainsi, les thèses codirigées se soutiendront obligatoirement en France ; celles qui feront l'objet de cotutelles pourront être soutenues sur place ou en France, en fonction des possibilités de constitution des jurys.

\section{La fin d'un partenariat?}

Ces quelques lignes ne sont pas conclusives, mais tirent les leçons de l'expérience. En effet, est apparue, dans les dernières années de cette action de coopération, une évolution forte de notre partenariat de recherche. Parti d'une formation à la recherche, par et pour la recherche, dispensée par les chercheurs français aux étudiants vietnamiens, ce partenariat est en voie de devenir aujourd'hui une aide à la création d'équipes de recherche et d'un corps d'habilités à diriger les recherches. De plus, les codirections d'étudiants, et a fortiori les cotutelles, vont exiger une cohérence et une entente scientifiques entre directeurs. Cela ne pourra se faire que si les personnes se choisissent elles-mêmes comme collaborateurs possibles dans la direction de recherche des doctorants... et dans l'intérêt de ces derniers. 
Ainsi, les relations entre partenaires se transforment en profondeur pour devenir des relations entre chercheurs ayant à négocier entre eux leurs intérêts de recherche. Du coup, des divergences d'intérêt scientifique apparaissent inévitablement et peuvent inciter nos partenaires à chercher auprès d'autres universités de nouvelles collaborations. De même que, de notre côté, des demandes de recherche peuvent être refusées, car trop éloignées de nos intérêts de recherche (et même de nos compétences de chercheurs).

Mais la fin de ce qui fut la raison d'être ensemble au départ n'est-elle pas la marque d'un partenariat incontestablement réussi ?

Régine Delamotte-Legrand

Laboratoire UMR 6065 DYALANG CNRS

Université de Rouen

regine.delamotte@univ-rouen.fr 